\title{
VARIABILIDADE ESPACIAL DA UMIDADE E DAS FRAÇÕES GRANULOMÉTRICAS DO SOLO EM UM PLANTIO DE BANANEIRAS IRRIGADO NO SEMIÁRIDO PERNAMBUCANO
}

\author{
Renato Augusto Soares Rodrigues ${ }^{1}$, Abelardo Antônio AssunÇão Montenegro ${ }^{1}$, \\ Daniella Pereira dos Santos ${ }^{1}$, Adriana Guedes Magalhães ${ }^{2}$, Robertson de Paiva Fontes Júnior ${ }^{1}$ \\ ${ }^{1}$ Universidade Federal Rural de Pernambuco ${ }^{2}$ Faculdade Santa Helena \\ $<$ renato.augustor@hotmail.com>, <abelardo.montenegro@yahoo.com.br> \\ <daniellapsantos@hotmail.com>.<rr_fontes@hotmail.com> \\ DOI: $10.21439 /$ conexoes.v11i3.884
}

\begin{abstract}
Resumo. A vegetação afeta significativamente a dinâmica de umidade do solo, tanto diretamente como por meio da interação com outros fatores, por exemplo, a textura do solo. A distribuição espacial da textura no terreno usualmente apresenta dependência espacial com a direção e a intensidade dos fluxos de água, que são dependentes das formas de relevo. Devido à importância da umidade e da textura do solo para a produção agrícola, principalmente em regiões semiáridas, o objetivo deste trabalho foi avaliar a variabilidade espacial da umidade e da textura do solo, em lote irrigado, na Bacia Experimental do Rio Ipanema, localizada no município de Pesqueira-PE. O estudo foi realizado na Fazenda Mimosa, localizada no distrito de Mimoso, município de Pesqueira, região agreste de Pernambuco. A umidade do solo foi medida utilizando um equipamento de "Time Domain Reflectometry" (TDR) em malha regular, espaçada de $8 \times 4 \mathrm{~m}$, totalizando 104 pontos. A amostragem de solo foi realizada em malha regular, espaçada de $8 \times 8 \mathrm{~m}$, totalizando 48 pontos. Os dados foram submetidos à análise estatística descritiva e geoestatística. O modelo de semivariograma que apresentou o melhor ajuste foi o exponencial para todas as variáveis analisadas. As variáveis estudadas apresentaram estrutura de dependência espacial, o que permitiu seu mapeamento, utilizando-se geoestatística. A estrutura de dependência espacial possibilitou observar a distribuição da umidade e da textura do solo, na área de estudo.
\end{abstract}

Palavras-chaves: Geoestatística. Manejo de água e solo. Dependência espacial.

\begin{abstract}
Vegetation significantly affects the soil moisture dynamics, either directly or through interaction with other factors, for example, soil texture. The spatial distribution of soil texture usually presents spatial dependence whit the direction and intensity of water flows, which are dependent on the landforms. Given the importance of moisture and soil texture for agricultural production, especially in semi-arid regions, the purpose of this study was to evaluate the spatial variability of moisture and soil texture in are irrigated plot the Experimental Basin of Ipanema River, located in the city of Pesqueira, Pernambuco. The study was conducted at Fazenda Mimosa, located in Mimoso district in the city of Pesqueira, agreste region of Pernambuco. Soil moisture was measured using Time Domain Reflectometry equipment in regular grid, at $8 \times 4 \mathrm{~m}$, comprising 104 points in total. Soil sampling was performed in regular grid, spaced at $8 \times 8$ meters, with 48 points in total. Data were submitted to the descriptive, statistical and geostatistics analysis. The semivariogram model that presented the best fit for all variables was the exponential. The variables presented spatial dependence structure, allowing its mapping. The spatial dependence structure allowed observation of the distribution of moisture and soil texture, in the studied area.
\end{abstract}

Keywords: Geostatistics. Management of water and soil. Spatial dependence.

\section{INTRODUÇÃO}

A umidade do solo é uma variável de relevância para a compreensão de uma série de processos hidrológicos e climáticos em diferentes escalas espaciais e temporais (BROCCA et al., 2009; HEATHMAN et al., 2009). Seus padrões espaciais são controlados por vários fato- 
VARIABILIDADE ESPACIAL DA UMIDADE E DAS FRAÇÕES GRANULOMÉTRICAS DO SOLO EM UM PLANTIO DE BANANEIRAS IRRIGADO NO SEMIÁRIDO PERNAMBUCANO

res, tais como topografia, propriedades do solo, tipo de vegetação, radiação solar, manejo da irrigação e precipitação (HÉBRARD et al. 2006, KORRES et al., 2010, RODRíGUEZ-ITURBE et al. 2006, ROSENBAUM et al. 2012; SVETLITCHNYI; PLOTNITSKIY; STEPOVAYA, 2003).

A umidade do solo interfere diretamente no crescimento das culturas e sua distribuição sofre forte influência do manejo da água em sistemas de cultivos agrícolas irrigados (SUN et al., 2009). As variações espaciais e temporais de umidade do solo podem ser minimizadas com o aumento da eficiência de irrigação, reduzindo os impactos ambientais e melhorando os rendimentos produtivos (KIM; EVANS; IVERSEN, 2008).

Faria et al. (2010) ressaltam a importância da irrigação para o adequado desenvolvimento da bananeira, que deve garantir a aplicação de água em quantidades apropriadas e em alta eficiência, de modo que a umidade do solo permita o pleno desenvolvimento da cultura. Oliveira, Coelho e Borges (2000) destacam que a deficiência hídrica causa grandes perdas à produção, reduz o crescimento apical e lateral do pseudocaule, diminui a área foliar, o número de pencas e de frutos por cacho. A adoção da irrigação permite não só ampliar o tempo de exploração da planta e o número de colheitas, como ainda melhorar a produção já existente.

Baroni et al. (2013) demonstraram que a vegetação afeta significativamente a dinâmica de umidade do solo, tanto diretamente como através da interação com outros fatores, por exemplo, a textura do solo.

A variação das características texturais do solo ocorrem em função da deposição de sedimentos, do relevo, do tempo de exposição dos materiais à ação do intemperismo, da vegetação (YOUNG; HAMMER, 2000) e principalmente do material de origem (CUNHA et al. 2005). A textura do solo também pode variar em função da estratigrafia da área de estudo (Marques Júnior; LEPSCH, 2000). Gobin, Campling e Feyen (2001) afirmam que a distribuição espacial da textura no terreno apresenta dependência espacial da direção e da intensidade dos fluxos de água, que são dependentes das formas de relevo. Assim, áreas de forma côncava condicionam movimento das partículas na direção perpendicular ao sentido da vertente. Já em segmento de forma convexa e de forma linear, visualiza-se a inversão dos movimentos na direção paralela ao sentido da vertente, conforme destaca Leão et al. (2010).

Portanto, devido à importância da umidade e da textura do solo para a produção agrícola, principalmente em regiões semiáridas, o objetivo deste trabalho foi avaliar a variabilidade espacial da umidade e das frações granulométricas do solo em lote irrigado, localizado na
Bacia Experimental do Rio Ipanema, localizada no município de Pesqueira-PE.

\section{MATERIAL E MÉTODOS}

O estudo foi realizado na Fazenda Mimosa, localizada no Distrito do Mimoso pertencente ao município de Pesqueira-PE, região do agreste de Pernambuco, com coordenadas de $08^{\circ} 10^{\prime} \mathrm{S}$ e $35^{\circ} 11^{\prime} \mathrm{W}$ e altitude de 650 m. A Fazenda Mimosa situa-se em um vale aluvial do Rio Ipanema, onde se desenvolve agricultura irrigada em pequena escala. O clima da região é do tipo BSsh (extremamente quente, semiárido) de acordo com a classificação de Köppen, com pluviometria média anual de $607 \mathrm{~mm}$ (MONTENEGRO; MONTENEGRO, 2006).

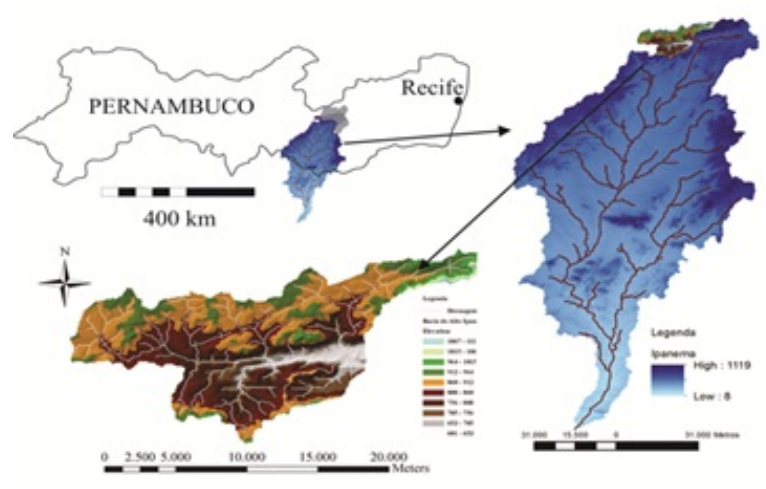

Figura 1: Mapa com a localização do município de Pesqueira com destaque para a Bacia do Rio Ipanema Fonte. Fonte: Fontes Júnior et al. (2012).

O solo da área de estudo é caracterizado como Neosolo Flúvico. Baseado no modelo de identificação de classes texturais do perfil do solo (triângulo textural) apresentada por Embrapa (2006), é possível classificar o perfil do solo com textura franco argilo arenosa. Suas características físicas e químicas estão apresentadas nas Tabelas 1 e 2, respectivamente.

Para a determinação da variável umidade do solo os ensaios foram conduzidos em uma área com malha regular, espaçada de $8 \mathrm{~m} \mathrm{x} 4 \mathrm{~m}$, formando um "grid" $96 \mathrm{~m}$ x $32 \mathrm{~m}$, totalizando 104 pontos amostrais, como mostra a Figura 2

A umidade do solo foi medida utilizando um equipamento de "Time Domain Reflectometry", TDR (Campbell ${ }^{\circledR}$, Hydrosense TM, Campbell Scientific, Australia), na camada de $0,00-0,20 \mathrm{~m}$. O estudo foi realizado nos dias 26, 27 e 28 de Novembro de 2014, período de estiagem na região, não ocorrendo precipitação pluviométrica para os dias de em que o estudo foi 
VARIABILIDADE ESPACIAL DA UMIDADE E DAS FRAÇÕES GRANULOMÉTRICAS DO SOLO EM UM PLANTIO DE BANANEIRAS IRRIGADO NO SEMIÁRIDO PERNAMBUCANO

Tabela 1: Características Físicas do solo do lote irrigado onde o trabalho foi desenvolvido

\begin{tabular}{ccccccc}
\hline $\begin{array}{c}\text { Profundidade } \\
(\mathrm{m})\end{array}$ & Areia & Argila & Silte & $\begin{array}{c}\text { Densidade } \\
\left(\mathrm{g} \mathrm{cm}^{-3}\right)\end{array}$ & $\begin{array}{c}\text { Porosidade } \\
\left(\mathrm{cm}^{3} \mathrm{~cm}^{-3}\right)\end{array}$ & $\begin{array}{c}\text { Condutividade Hidráulica } \\
\left(\mathrm{mm} \mathrm{s}^{-1}\right)\end{array}$ \\
\hline $0-0,20$ & 587,6 & 240,5 & 171,9 & 1,47 & 0,52 & 0,183 \\
\hline
\end{tabular}

Tabela 2: Características Químicas do solo do lote irrigado onde o trabalho foi desenvolvido

\begin{tabular}{ccccccc}
\hline \multirow{2}{*}{$\begin{array}{c}\text { Profundidade } \\
(\mathrm{m})\end{array}$} & $\mathrm{P},\left(\mathrm{mg} \mathrm{dm}^{-3}\right)$ & $\mathrm{pH}\left(\mathrm{H}_{2} \mathrm{O}\right)$ & $\mathrm{K}$ & $\mathrm{Al}$ & $\mathrm{Ca}$ & $\mathrm{Mg}$ \\
\cline { 4 - 7 } & $>40$ & 6,80 & & \multicolumn{4}{c}{$\mathrm{Cmolc} \mathrm{dm}^{-3}$} & \\
\hline $0-0,20$ & $>0.45$ & 0,00 & 7,10 & 0,50 \\
\hline
\end{tabular}

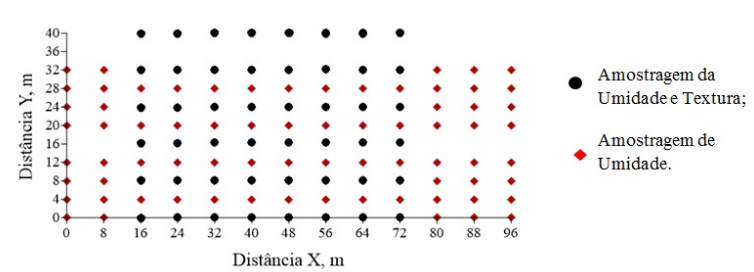

Figura 2: Localização dos pontos de amostragem na área experimental para variável umidade (\%) e textura do solo.

realizado. Foram realizadas 4 medições de umidade do solo, das quais duas foram realizadas antes da irrigação as 8 horas e 9 horas da manhã e duas foram realizadas após a irrigação as 12 horas e 16 horas, sendo a irrigação realizada no período de 10 horas às 11 horas e 30 minutos.

No plantio de bananeiras foi utilizada a variedade "Prata", com espaçamento $4 \mathrm{~m} \mathrm{x} 4 \mathrm{~m}$, adotando como manejo da cultura a aplicação de resíduos de pseudocaule e folhas sobre a superfície do solo. No momento do estudo o plantio estava em fase de produção. O sistema de irrigação adotado é do tipo aspersão convencional, com aspersores sub-copa e espaçamento entre aspersores de $6 \mathrm{~m}$ x $12 \mathrm{~m}$, com intensidade de aplicação de $8,90 \mathrm{~mm} / \mathrm{h}$, lâmina média aplicada de 7,7 mm, com turno de rega diário e tempo de irrigação de 1 hora e 30 minutos, pressão de serviço de 20 mca e diâmetro de alcance de $21,2 \mathrm{~m}$, cujo coeficiente de uniformidade de aplicação (CUC), proposto por Christiansen (1942) é de 75,42\%. Bernardo, Soares e Mantovani (2006) afirmam que o valor de CUC considerado como o mínimo aceitável é de $70 \%$.

Para a determinação das frações granulométricas do solo as coletas foram realizadas em uma subárea, utilizada para a medição da umidade, sendo a malha regular, espaçada de $8 \mathrm{~m} \mathrm{x} 8 \mathrm{~m}$, formando um "grid" $56 \mathrm{~m} \mathrm{x} 40$ $\mathrm{m}$, totalizando 48 pontos amostrais, como mostra a Figura 2

As frações areia, argila e silte foram determinadas pelo método do densímetro de Boyoucus, conforme metodologia proposta por Embrapa (1997).

A estatística descritiva foi utilizada para avaliar a média, a mediana, o desvio padrão, os coeficientes de assimetria e curtose e o coeficiente de variação, para obter informações sobre a dispersão e a distribuição das variáveis, e a aderência à distribuição normal, segundo o teste de Kolmogorov-Smirnov, ao nível de 5\% de significância.

A análise da dependência espacial foi conduzida por meio da geoestatística. Com base na hipótese de estacionariedade, a autocorrelação espacial entre locais vizinhos foi calculada através da semivariância $\gamma(\mathrm{h})$, sendo "h" a distância de separação entre os pontos.

Após a obtenção dos semivariogramas experimentais, por meio do software GEO-EAS, foram testados os modelos gaussiano, esférico, exponencial e linear. Do ajuste de um modelo matemático aos valores calculados, foram estimados os coeficientes do modelo teórico para o semivariograma (o efeito pepita, $\mathrm{C}_{0}$; patamar, $\mathrm{C}_{0}+\mathrm{C}_{1}$ e o alcance, a).

Foi escolhido o modelo que apresentou o melhor ajuste aos valores experimentais, bem como erros padronizados com média próxima a zero e desvio padrão próximo a um, segundo a técnica de validação cruzada de Jack-Knifing, detalhada Montenegro e Montenegro (2006).

No que diz respeito à construção dos mapas de isolinhas, utilizando o algoritmo da krigagem, foi adotado o programa computacional Surfer 9.0 (GOLDEN, 2010).

\section{RESULTADOS E DISCUSSÃO}

$\mathrm{Na}$ Tabela 3 têm-se as medidas de posição e dispersão para as variáveis umidade do solo e Frações granulométricas. O coeficiente de variação $(\mathrm{CV})$ de acordo com a classificação utilizada por Warrick (1998) consideram que a umidade do solo apresenta média variabilidade em todos os instantes avaliados. Este fato pode ser explicado pelo fato de que os valores de média, mediana, desvio padrão e variância apresentaram baixas varia- 
VARIABILIDADE ESPACIAL DA UMIDADE E DAS FRAÇÕES GRANULOMÉTRICAS DO SOLO EM UM PLANTIO DE BANANEIRAS IRRIGADO NO SEMIÁRIDO PERNAMBUCANO

ções entre os instantes de medição de umidade como pode ser visto na tabela 1 . Outro fator que explica esse comportamento dos dados é o fato deles terem obedecido a distribuição normal de frequências, comprovado pelo teste de Kolmogorov Smirnov ao nível de 5\% de significância.

Souza, Montenegro e Freire (2008), avaliando a variabilidade espacial da umidade do solo em um Neossolo Fluvico, no mesmo perímetro irrigado deste estudo, encontraram resultados semelhantes usando uma malha regular de $5 \mathrm{~m}$ x $5 \mathrm{~m}$, com 101 pontos de amostragem. Grego et al. (2006), avaliando a variabilidade espacial da umidade do solo em um Latossolo Vermelho, encontraram resultados semelhantes usando uma malha regular de $10 \mathrm{~m}$ x $20 \mathrm{~m}$, com 101 pontos de amostragem.

As frações areia e agila apresentaram baixa variabilidade, enquanto que o valor de CV para a variável silte apresentou média variabilidade, na camada estudada. Os valores de CV encontrados neste estudo corroboram com os observados com Andrade et al. (2014), que estudaram a variabilidade espacial das classes texturais em Neossolo Flúvico e obtiveram resultados semelhantes, ou seja, baixa variabilidade para as frações areia e argila, enquanto a fração silte apresenta média variabilidade, para a camada $0,00-0,20 \mathrm{~m}$. A maior variabilidade, para a fração silte pode estar relacionada com a sua maior mobilidade no solo de um vale aluvial de rio intermitente, conforme afirmado por Montenegro e Montenegro (2006). O teste de Kolgomorov Smirnov indicou normalidade dos dados ao nível de $5 \%$ de significância para a areia, a argila e o silte.

Analisando-se o gráfico de "box-plot" (Figura 3) para as variáveis, a umidade nos instantes U1 e U2 (antes da irrigação) apresentaram baixa assimetria negativa. Nos instantes U3 e U4 também houve assimetria negativa, porém a maior dispersão dos dados foi observada para os valores abaixo do quartil inferior. Este fato pode ser explicado pelos valores obtidos na avaliação do sistema de irrigação, onde obteve-se $72,30 \%$ e $75,42 \%$ para o Coeficiente de Uniformidade de Distribuição (CUD) e o Coeficiente de Uniformidade de Christiansen (CUC), respectivamente. Segundo a classificação de Mantovani (2001), o resultado obtido para o CUD é considerado bom, enquanto que o resultado para CUC é considerado razoável. Dessa forma, os resultados obtidos com o experimento realizado indicam que o sistema de irrigação tem uma aceitável uniformidade de distribuição de água. Após a avaliação da uniformidade da umidade do solo observou-se que para o Neossolo Flúvico estudado a irrigação, ocasionou CUD de $74,40 \%$ e CUC de $77,12 \%$.

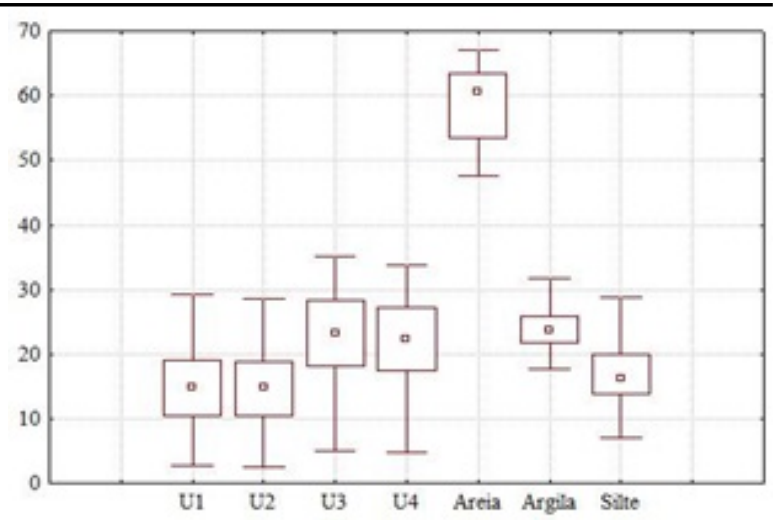

Figura 3: Gráfico "Box-Plot" para as variáveis U1, U2, U3, U4, Areia, Silte e Argila.

U1 - primeira medição de umidade do solo (\%); U2 - segunda medição de umidade de solo (\%); U3 - terceira medição de umidade do solo (\%); e U4 - quarta medição de umidade do solo (\%).

Para os teores de areia, argila e silte, verifica-se assimetria para as 3 variáveis. Quanto aos valores de argila e silte, pode ser notada a ocorrência de uma dispersão maior nos dados para os valores acima do quartil inferior, ou seja, assimetria positiva. Para areia observa-se o comportamento oposto, sendo a dispersão dos dados para os valores abaixo do quartil inferior, e os dados apresentam assimetria negativa.

Os semivariogramas experimentais mostraram a existência de uma estrutura de dependência espacial, a qual pode ser descrita por meio de um modelo exponencial para os 4 instantes de avaliação da umidade do solo e para a textura do solo, que produziu o melhor ajuste, e apresentou pela técnica da validação cruzada o desvio padrão do erro reduzido mais próximo de 1 e a média mais próxima a 0 . As variáveis, o modelo ajustado e os parâmetros do modelo são apresentados na Tabela 1] A Figura 4 apresenta os modelos ajustados e as semivariâncias experimentais.

O modelo exponencial apresentou alcances de 3,90 e 3,30 m para os instantes de medição antes da irrigação e 7,10 e 7,88 m após a irrigação, ou seja, a irrigação produziu um aumento superior a $100 \%$ na escala de dependência espacial. Logo, a irrigação influencia fortemente na dependência espacial da umidade do solo. Verifica-se inclusive que o grau de dependência espacial aumenta nos instantes que sucedem a irrigação. Observa-se que os dados para U1 e U2 são mais uniformes, enquanto que para U3 e U4 os semivariogramas se apresentam mais estruturados.

Brocca et al. (2009), estudando a variabilidade espacial da umidade do solo, utilizando TDR na camada de 
VARIABILIDADE ESPACIAL DA UMIDADE E DAS FRAÇÕES GRANULOMÉTRICAS DO SOLO EM UM PLANTIO DE BANANEIRAS IRRIGADO NO SEMIÁRIDO PERNAMBUCANO

Tabela 3: Estatística descritiva, parâmetros de ajuste e de validação dos semivariogramas para as variáveis umidade e frações granulométricas do solo

\begin{tabular}{|c|c|c|c|c|c|c|c|}
\hline Estatística descritiva & U1 & $\mathrm{U} 2$ & U3 & U4 & Areia & Argila & Silte \\
\hline $1^{\circ}$ Quartil & 19,1 & 18,75 & 28,4 & 27,26 & 63,44 & 25,76 & 21,71 \\
\hline Mediana & 14,85 & 14,95 & 23,25 & 22,32 & 59,38 & 23,76 & 16,24 \\
\hline Média & 14,85 & 14,62 & 22,59 & 21,69 & 58,63 & 24,17 & 17,18 \\
\hline $3^{\circ}$ Quartil & 10,45 & 10,37 & 18,15 & 17,42 & 53,43 & 21,76 & 14,31 \\
\hline Coeficiente de Assimetria & $-0,05$ & $-0,09$ & $-0,46$ & $-0,46$ & $-0,53$ & 0,31 & 0,13 \\
\hline Coeficiente de Curtose & $-0,5$ & $-0,54$ & $-0,46$ & $-0,46$ & $-0,37$ & $-0,42$ & $-0,54$ \\
\hline Desvio padrão & 5,98 & 5,87 & 7,28 & 6,99 & 5,89 & 3,49 & 5,36 \\
\hline Variância & 35,78 & 34,51 & 53,08 & 48,92 & 34,72 & 12,24 & 28,74 \\
\hline Coeficiente de variação (\%) & 40,26 & 40,15 & 32,22 & 36,22 & 10 & 14,43 & 30,66 \\
\hline $\mathrm{N}^{\mathrm{o}}$ de elementos da amostra & 104 & 104 & 104 & 104 & 48 & 48 & 48 \\
\hline \multicolumn{8}{|c|}{ Parâmetros de Ajuste dos Semivariogramas } \\
\hline Efeito Pepita $\left(\mathrm{C}_{0}\right)$ & 0,49 & 3,3 & 0,46 & 0,47 & 0,29 & 0,65 & 5,17 \\
\hline Patamar $\left(\mathrm{C}_{0}+\mathrm{C}_{1}\right)$ & 35,04 & 29,07 & 52,67 & 50,74 & 24,37 & 9,17 & 22,03 \\
\hline Alcance & 3,9 & 3,3 & 7,1 & 7,88 & 5,2 & 4,81 & 6,17 \\
\hline Grau de dependência $\left[\mathrm{C}_{0} /\left(\mathrm{C}_{0}+\mathrm{C}_{1}\right)\right] \times 100$ & 1,39 & 11,35 & 0,87 & 0,92 & 1,18 & 7,08 & 23,46 \\
\hline Modelo & Exp. & Exp. & Exp. & Exp. & Exp. & Exp. & Exp. \\
\hline \multicolumn{8}{|c|}{ Parâmetros de Validação dos Semivariogramas } \\
\hline Média & 0,012 & 0,019 & 0,028 & 0,098 & $-0,008$ & $-0,017$ & 0,016 \\
\hline Desvio Padrão & 0,987 & 0,991 & 1,012 & 1,002 & 1,021 & 1,015 & 0,991 \\
\hline
\end{tabular}

U1 - primeira medição de umidade do solo (\%); U2 - segunda medição de umidade de solo (\%); U3 - terceira medição de umidade do solo (\%); e U4 - quarta medição de umidade do solo (\%).

0-0,20 m, na região do Vale do Tibre, na Itália, região de clima mediterrânico e precipitação média anual de aproximadamente $900 \mathrm{~mm}$, observaram que o modelo exponencial produziu o melhor ajuste; entretanto, Western et al. (2004) estudando uma bacia hidrográfica com características semiáridas testaram o ajuste aos modelos teóricos e verificaram que o modelo exponencial não apresenta desempenho satisfatório no comportamento da variabilidade espacial da umidade do solo. Cichota, Hurtado e Lier (2006), analisando o padrão espacial da umidade do solo em condições tropicais, constataram que o modelo que melhor descreve o comportamento espacial da umidade do solo é o Gaussiano.

O modelo que melhor se ajustou aos dados granulométricos foi o exponencial, apresentando maior continuidade na distribuição das variáveis na camada mais superficial da área estudada, concordando com estudo de (SOUZA; QUEIROZ; GHEYI, 2000), trabalhando com malhas regulares espaçadas em $50 \mathrm{~m}$.

Os alcances encontrados neste trabalho foram de $5,2,4,81$ e $6,17 \mathrm{~m}$ para areia, argila e silte respectivamente. Os maiores alcances foram observados para silte e areia, respectivamente, demonstrando que estes atributos, são os que apresentam maiores continuidades espaciais, possibilitando melhor precisão nas estimativas em locais não amostrados. De acordo com os critérios propostos por Cambardella et al. (1994), baixos valores de efeito pepita representam uma forte dependência espacial nas variáveis avaliadas, como observado nos resultados. Isso explica elevados graus de dependência espacial para as variáveis observadas nesse estudo, exceto para a fração granulométrica silte que apresentou moderada dependência espacial, devido seu elevado efeito pepita $(5,17)$ e baixo valor de patamar $(22,03)$. O lote irrigado em que o estudo foi desenvolvido pertence a um vale aluvial, e a dependência espacial do silte pode está associada a processos geomorfológicos, já que ocorrem pela acumulação e distribuições desuniformes de sucessivos depósitos de materiais oriundos de outros lugares, proporcionados pela posição topográfica que ocupam, além do manejo a que são submetidos (MONTENEGRO; MONTENEGRO, 2006).

Souza, Montenegro e Freire (2008), trabalhando em Neossolo Flúvico, em um lote irrigado da Fazenda Nossa Senhora do Rosário, cultivado com cenoura, obtiveram alcances de 56, 50 e $55 \mathrm{~m}$ para areia, argila e silte respectivamente, e melhor ajuste ao modelo gaussiano. Já Eguchi, Silva e Oliveira (2002), trabalhando em encosta de um vale aluvial, encontraram alcances de $15,49,5$ e $14,5 \mathrm{~m}$ para areia, argila e silte, e atribuíram 


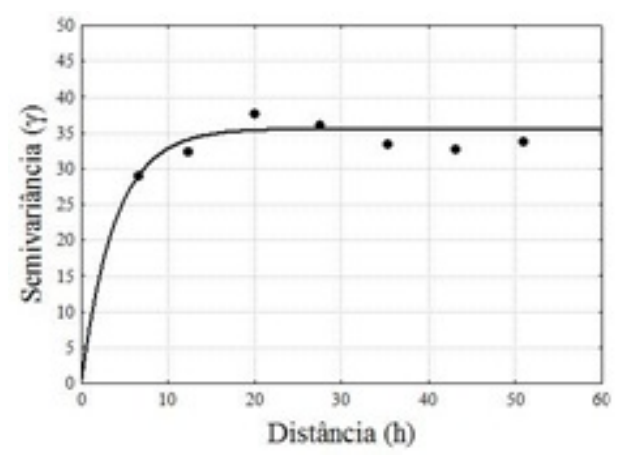

(a)

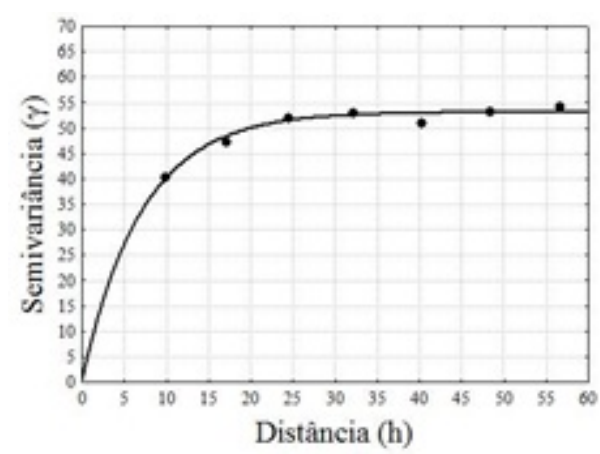

(c)

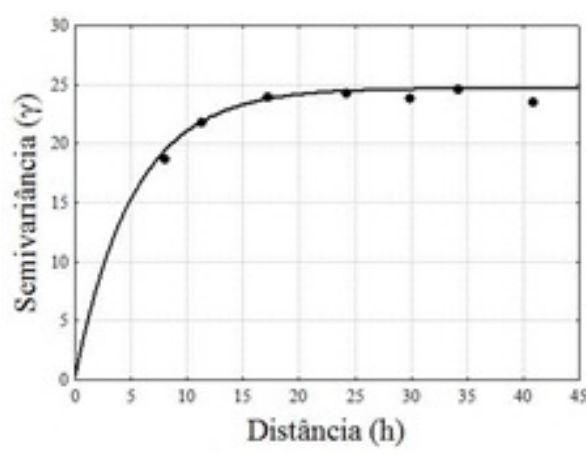

(e)

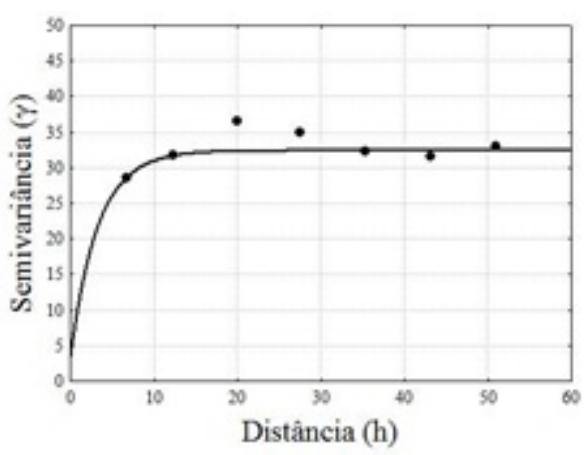

(b)

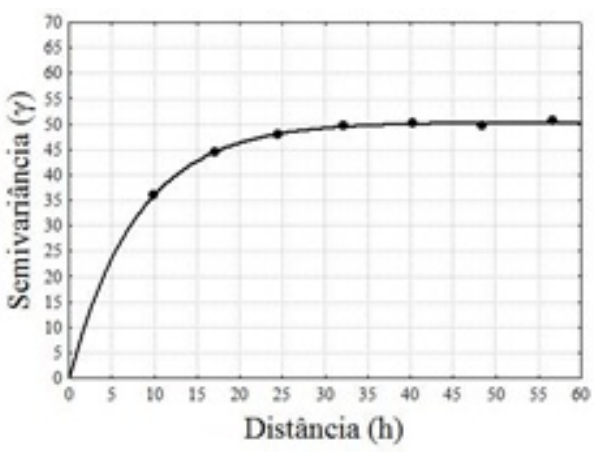

(d)

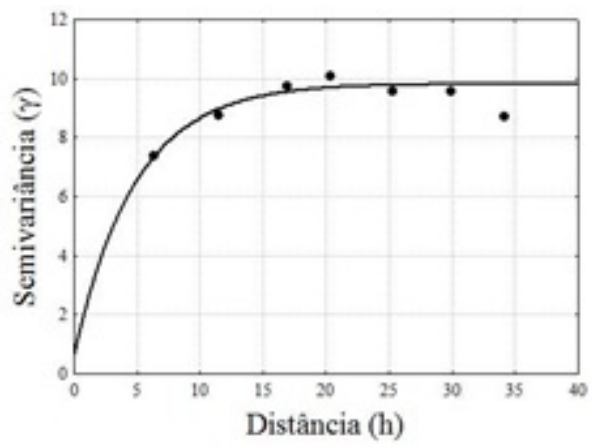

(f)

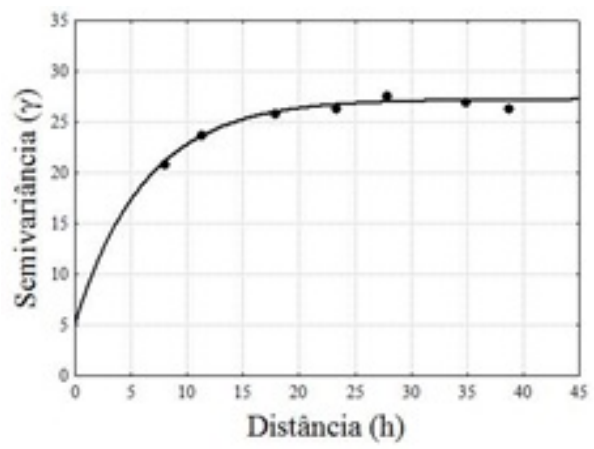

(g)

Figura 4: Semivariogramas ajustados para as variáveis umidade (\%) no momento U1 (a); momento U2 (b); momento U3 (c); momento U4 (d); e textura do solo: teor de areia (g.kg-1) (e); teor de argila $\left(\mathrm{g} . \mathrm{kg}^{-1}\right)$ (f); teor de silte $\left(\mathrm{g} . \mathrm{kg}^{-1}\right)(\mathrm{g})$, na profundidade $0,00-0,20 \mathrm{~m}$.

Conex. Ci. e Tecnol. Fortaleza/CE, v. 11, n. 3, p. 134 - 143, nov. 2017 
VARIABILIDADE ESPACIAL DA UMIDADE E DAS FRAÇÕES GRANULOMÉTRICAS DO SOLO EM UM PLANTIO DE BANANEIRAS IRRIGADO NO SEMIÁRIDO PERNAMBUCANO

os resultados principalmente às contribuições presentes na formação do solo.

Os mapas de isolinhas da umidade do solo (Figura 5- a, b, c e d) antes e após a irrigação, para a camada de 0-0,20 m, apresentam diminuição na variabilidade da umidade, e umidade máxima de $22 \%$ no início da área, uma média em toda a área de $16 \%$ e, nas áreas centrais, uma diminuição da umidade, chegando a valores de umidade de $10 \%$. Após a irrigação, existe uma variação do conteúdo de água na camada de 0 - 0,20 $\mathrm{m}$ do perfil do solo em toda a área, com valor máximo de $31 \%$, porém o conteúdo de água nas áreas centrais continua sendo menor que a umidade nas extremidades, chegando a valores mínimos de umidade de $13 \%$. Os mapas de umidade mostram que as regiões centrais possuem menor conteúdo de água, indicando que o movimento de água nessa área ocorre a partir do centro para as extremidades.

As áreas mais escuras no mapa de isolinhas (Figura 5- a, b, c e d) apresentam maiores valores de umidade do solo. Souza et al. (2008) afirmam que os mapas de isolinhas são de grande importância, pois de posse dessas informações pode-se examinar as regiões sistematicamente com baixa umidade, e daí verificar o sistema de irrigação ou a existência de uma possível "mancha de solo" presente. Alves et al. (2011), trabalhando em malha amostral semelhante $(10 \mathrm{~m}$ x $24 \mathrm{~m})$, utilizaram mapas de isolinhas para interpretar os resultados de medição de umidade do solo vinte e quatro horas após a irrigação, obtiveram CUC e CUD de 76,69\% e 72,22\%, respectivamente e observaram que o modelo linear produziu melhor ajuste. O grau de dependência espacial diminui devido ao aumento do patamar ser consideravelmente maior em relação ao aumento do efeito pepita para a medição da umidade 24 horas após a irrigação.

Observa-se que o local com maiores valores de umidade está localizado na porção inferior do lado esquerdo das imagens, o que pode ser devido ao sentido da declividade do terreno e à desuniformidade do sistema de irrigação, nesse setor. Este padrão manteve-se durante todos os momentos de medição, o que é indicativo de uma possível dependência temporal. Coelho Filho, Coelho e Gonçalves (2001), observaram resultados semelhantes, ao avaliar a variabilidade espacial da umidade em um lote irrigado por microaspersão no estado de São Paulo encontrando maior disponibilidade de água na parte baixa da superfície, em direção à coordenada $\mathrm{X}$ e, para a região superior do terreno, observaram baixos valores de água armazenada.

Verifica-se que a distribuição superficial da areia registra seus menores teores na posição inferior da imagem (Figura 5 (e)). Contrastando com a distribuição observada para os teores de argila e de silte, que se distribuem em maior quantidade nas áreas em que o teor de areia é menor. Esses resultados também foram encontrados por Leão et al. (2010), que avaliaram a variabilidade espacial das frações granulométricas do solo de uma encosta de um vale aluvial no estado de São Paulo.

\section{CONCLUSÕES}

Verifica-se que as variáveis analisadas obedecem à distribuição normal de frequência e que possuem uma variabilidade média. A umidade do solo apresentou média variabilidade e alta dependência espacial, para todos os instantes de avaliação. As frações granulométricas areia e argila apresentaram baixa variabilidade e alta dependência espacial, enquanto que o Silte apresentou média variabilidade e moderada dependência espacial. Por meio dos mapas de isolinhas, observa-se que as regiões centrais possuem menor conteúdo de água, indicando que o movimento de água nessa área ocorre a partir do centro para as extremidades. A irrigação promoveu significativo aumento na variabilidade e na dependência espacial da umidade do solo.

\section{AGRADECIMENTO}

Ao CNPq e Finep-REHIDRO pelo suporte na realização deste estudo.

\section{REFERÊNCIAS}

ALVES, W. W. A.; NETO, J. D.; MATOS, J. d. A. de; AZEVEDO, C. A. V. de; PORDEUS, R. V. Análise geoestatística da distribuição de água no solo aplicada por sistema de irrigação localizada. Revista Caatinga, v. 24, n. 2, p. 143-151, 2011.

ANDRADE, c. w. 1.; SILVA, j. s.; MONTENEGRO, a. a. a.; MONTENEGRO, s. m. g. 1.; TAVARES, u. e. Variabilidade espacial da textura em solo aluvial sob o cultivo de cenoura (daucus carota 1.). Revista Pernambucana de Tecnologia, v. 2, n. 1, p. 59-67, 2014.

BARONI, G.; ORTUANI, B.; FACCHI, A.; GANDOLFI, C. The role of vegetation and soil properties on the spatio-temporal variability of the surface soil moisture in a maize-cropped field. Journal of Hydrology, Elsevier, v. 489, n. 1, p. 148-159, 2013.

BERNARDO, s.; SOARES, a.; MANTOVANI, e. Manual de Irrigação. Viçosa, MG: UFV, 2006. 148-159 p. 


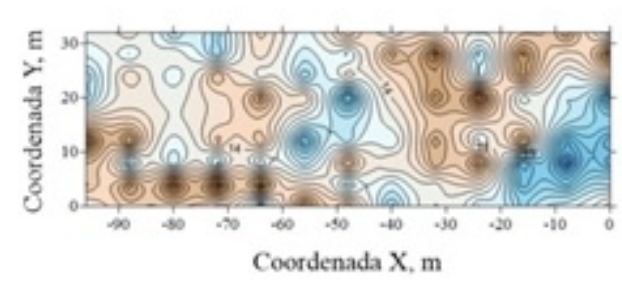

(a)

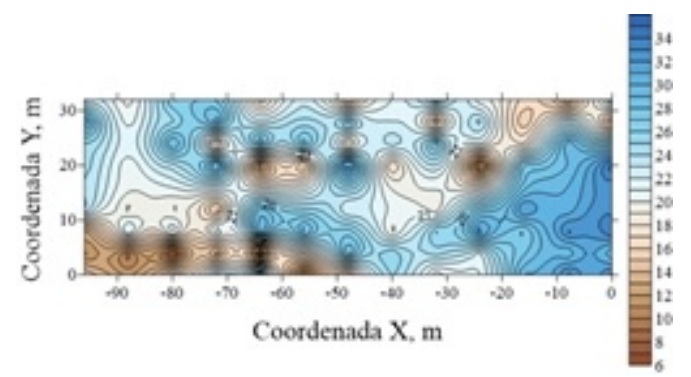

(c)

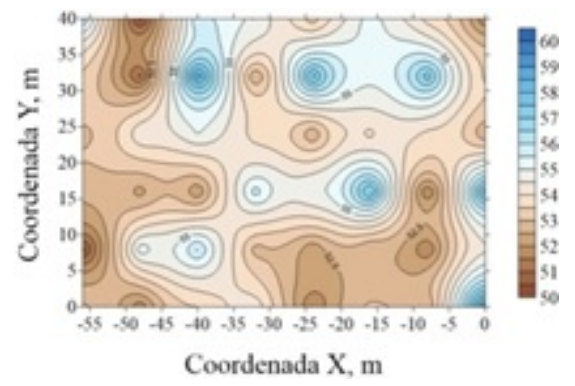

(e)

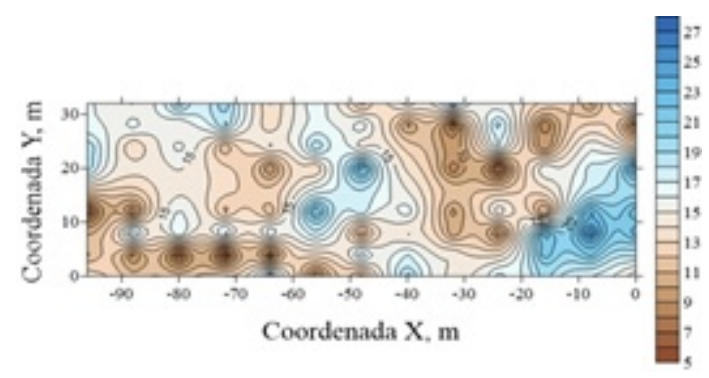

(b)

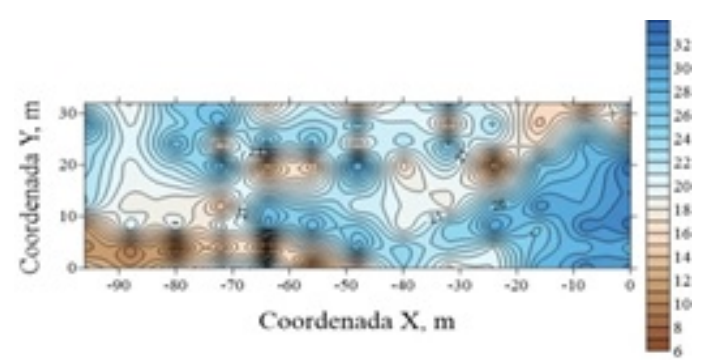

(d)

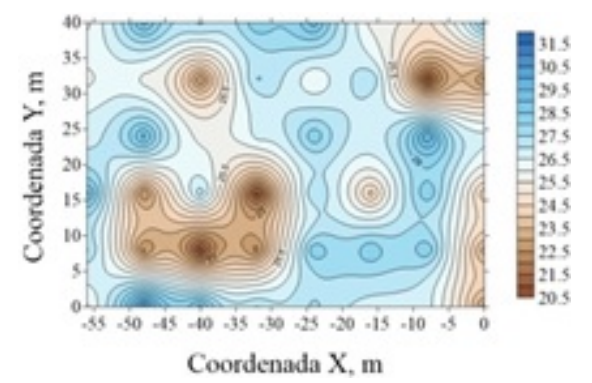

(f)

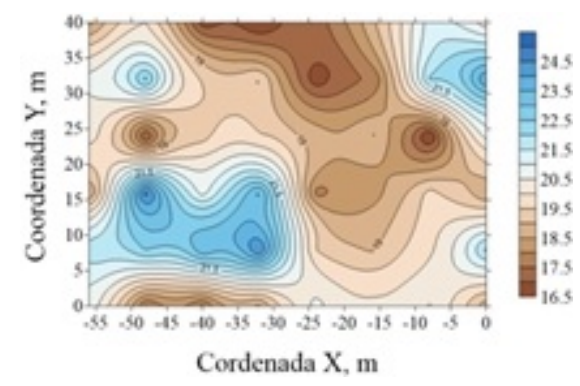

(g)

Figura 5: Mapa de Isolinhas para as variáveis umidade (\%) no momento U1 (a); momento U2 (b); momento U3 (c); momento U4 (d); e textura do solo: teor de areia $\left(\mathrm{g} \cdot \mathrm{kg}^{-1}\right)(\mathrm{e})$; teor de argila $\left(\mathrm{g} \cdot \mathrm{kg}^{-1}\right)(\mathrm{f})$; teor de silte $\left(\mathrm{g} \cdot \mathrm{kg}^{-1}\right)(\mathrm{g})$, na profundidade $0,00-0,20 \mathrm{~m}$. 
VARIABILIDADE ESPACIAL DA UMIDADE E DAS FRAÇÕES GRANULOMÉTRICAS DO SOLO EM UM PLANTIO DE BANANEIRAS IRRIGADO NO SEMIÁRIDO PERNAMBUCANO

BROCCA, L.; MELONE, F.; MORAMARCO, T.; MORBIDELLI, R. Soil moisture temporal stability over experimental areas in central italy. Geoderma, Elsevier, v. 148, n. 3, p. 364-374, 2009.

CAMBARDELLA, C. et al. Field-scale variability of soil properties in central iowa soils. Soil science society of America journal, Soil Science Society of America, v. 58, n. 5, p. 1501-1511, 1994.

CHRISTIANSEN, J. Irrigation by sprinkling. berkeley: University of california agricultural experiment station. Bulletin, v. 1, n. 670, p. 124, 1942.

CICHOTA, R.; HURTADO, A. L. B.; LIER, Q. d. J. V. Spatio-temporal variability of soil water tension in a tropical soil in brazil. Geoderma, Elsevier, v. 133, n. 3, p. 231-243, 2006.

Coelho Filho, M. A.; COELHO, R. D.; GONÇALVES, A. C. Variabilidade espacial de variáveis físico-hídricas do solo em um pomar de lima ácida tahiti, irrigado por microaspersão. Revista Brasileira de Engenharia Agrícola e Ambiental, Departamento de Engenharia Agrícola-UFCG, v. 5, n. 2, p. 239-246, 2001.

CUNHA, P.; JÚNIOR, J. M.; CURI, N.; PEREIRA, G. T.; LEPSCH, I. F. Superfícies geomórficas e atributos de latossolos em uma sequiência areníticobasaltica da região de jaboticabal (sp). Revista Brasileira de Ciência do Solo, Sociedade Brasileira de Ciência do Solo, v. 29, n. 1, p. 81-90, 2005.

EGUCHI, E. S.; SILVA, E. L. d.; OLIVEIRA, M. S. d. Variabilidade espacial da textura e da densidade de partículas em um solo aluvial no município de lavras, mg. Revista Brasileira de Engenharia Agrícola e Ambiental, Departamento de Engenharia Agrícola-UFCG, v. 6, n. 2, p. 242-246, 2002.

EMBRAPA. Manual de métodos de análise de solo. 2. ed. Rio de Janeiro, 1997. 212 p.

Sistema brasileiro de classificação de solos. 2. ed. Rio de Janeiro, 2006.

FARIA, H. C. d.; DONATO, S. L. R.; PEREIRA, M. C. T.; SILVA, S. d. O. Avaliação fitotecnica de bananeiras tipo terra sob irrigação em condições semi-áridas. Ciência e Agrotecnologia, SciELO Brasil, v. 34, n. 4, p. 830-836, 2010.

Fontes Júnior; MONTENEGRO, A. A. A.; MONTENEGRO, S. M. G. L.; SANTOS, T. E. M.

D. Estabilidade temporal da potenciometria e da salinidade em vale aluvial no semiárido de pernambuco. Revista Brasileira de Engenharia Agricola e Ambiental-Agriambi, v. 16, n. 11, p. 1188-1197, 2012.

GOBIN, A.; CAMPLING, P.; FEYEN, J. Soillandscape modelling to quantify spatial variability of soil texture. Physics and Chemistry of the Earth, Part B: Hydrology, Oceans and Atmosphere, Elsevier, v. 26, n. 1, p. 41-45, 2001.

GOLDEN, S. "Surfer version 9.0". Colorado: [s.n.], 2010. Disponível em: <www.goldensoftware.com>

GREGO, C. R.; VIEIRA, S. R.; ANTONIO, A. M.; ROSA, S. C. D. Geostatistical analysis for soil moisture content under the no tillage cropping system. Scientia Agricola, SciELO Brasil, v. 63, n. 4, p. 341-350, 2006.

HEATHMAN, G. C.; LAROSE, M.; COSH, M. H.; BINDLISH, R. Surface and profile soil moisture spatio-temporal analysis during an excessive rainfall period in the southern great plains, usa. Catena, Elsevier, v. 78, n. 2, p. 159-169, 2009.

HÉBRARD, O.; VOLTZ, M.; ANDRIEUX, P.; MOUSSA, R. Spatio-temporal distribution of soil surface moisture in a heterogeneously farmed mediterranean catchment. Journal of Hydrology, Elsevier, v. 329, n. 1, p. 110-121, 2006.

KIM, Y.; EVANS, R. G.; IVERSEN, W. M. Remote sensing and control of an irrigation system using a distributed wireless sensor network. IEEE transactions on instrumentation and measurement, IEEE, v. 57, n. 7, p. 1379-1387, 2008.

KORRES, W.; KOYAMA, C.; FIENER, P.; SCHNEIDER, K. Analysis of surface soil moisture patterns in agricultural landscapes using empirical orthogonal functions. Hydrology and Earth System Sciences, Copernicus GmbH, v. 14, n. 5, p. 751-764, 2010.

LEÃO, M. G.; JÚNIOR, J. M.; SOUZA, Z. M. d.; PEREIRA, G. T. Variabilidade espacial da textura de um latossolo sob cultivo de citros. Ciência e Agrotecnologia, Editora da Universidade Federal de Lavras (UFLA), v. 34, n. 1, p. 121-131, 2010.

MANTOVANI, E. AVALIA: Programa de avaliação da irrigação por aspersão e localizada. Viçosa, MG, 2001.

Marques Júnior, J.; LEPSCH, I. F. Depósitos superficiais neocenozóicos, superfícies geomórficas e solos em monte alto, sp. Geociências, Universidade Estadual Paulista, v. 19, n. 2, p. 265-282, 2000. 
VARIABILIDADE ESPACIAL DA UMIDADE E DAS FRAÇÕES GRANULOMÉTRICAS DO SOLO EM UM PLANTIO DE BANANEIRAS IRRIGADO NO SEMIÁRIDO PERNAMBUCANO

MONTENEGRO, A. A.; MONTENEGRO, S.

Variabilidade espacial de classes de textura, salinidade e condutividade hidráulica de solos em planície aluvial. Revista Brasileira de Engenharia Agrícola e Ambiental, SciELO Brasil, v. 10, n. 1, p. 30-37, 2006.

OLIVEIRA, S. d. 1.; COELHO, E. F.; BORGES, A. I. Irrigação e fertirrigação. In: . [S.1.]: Embrapa

Comunicação para Transferência de Tecnologia, 2000. cap. Banana: produção, aspectos técnicos, p. 60-72.

RODRíGUEZ-ITURBE, I.; ISHAM, V.; COX, D. R.; MANFREDA, S.; PORPORATO, A.

Space-time modeling of soil moisture: Stochastic rainfall forcing with heterogeneous vegetation.

Water Resources Research, v. 42, n. 6, p. n/a-n/a, 2006. ISSN 1944-7973. W06D05. Disponível em: <http://dx.doi.org/10.1029/2005WR004497>.

ROSENBAUM, U. et al. Seasonal and event dynamics of spatial soil moisture patterns at the small catchment scale. Water Resources Research, v. 48, n. 10, p. n/a-n/a, 2012. ISSN 1944-7973. W10544. Disponível em: <http://dx.doi.org/10.1029/2011WR011518>.

SOUZA, E. R.; MONTENEGRO, A. A.; FREIRE, M. B. d. S. Evolução e variabilidade espacial da salinidade em neossolo flúvico cultivado com cenoura sob irrigação. R. Bras. Eng. Agríc. Ambiental, v. 12, n. 6, p. 584-592, 2008.

SOUZA, E. R. et al. Variabilidade espacial das frações granulométricas e da salinidade em um neossolo flúvico do semi-árido. Ciência Rural, SciELO Public Health, v. 38, n. 3, p. 698-704, 2008.

SOUZA, L. d.; QUEIROZ, J.; GHEYI, H. Variabilidade espacial da salinidade de um solo aluvial no semi-árido paraibano. Revista Brasileira de Engenharia Agrícola e Ambiental, v. 4, n. 1, p. 35-40, 2000 .

SUN, Y. et al. A solar-powered wireless cell for dynamically monitoring soil water content. Computers and Electronics in Agriculture, Elsevier, v. 69, n. 1, p. 19-23, 2009.

SVETLITCHNYI, A.; PLOTNITSKIY, S.;

STEPOVAYA, O. Spatial distribution of soil moisture content within catchments and its modelling on the basis of topographic data. Journal of Hydrology, Elsevier, v. 277, n. 1, p. 50-60, 2003.

WARRICK, A. Spatial variability of soil physical properties in the field. In: [S.1.]: Academic Press, 1998. cap. Environmentalsoil physics, p. 655-675.
WESTERN, A. W. et al. Spatial correlation of soil moisture in small catchments and its relationship to dominant spatial hydrological processes. Journal of Hydrology, Elsevier, v. 286, n. 1, p. 113-134, 2004.

YOUNG, F.; HAMMER, R. Defining geographic soil bodies by landscape position, soil taxonomy, and cluster analysis. Soil Science Society of America Journal, Soil Science Society, v. 64, n. 3, p. 989-998, 2000 . 